
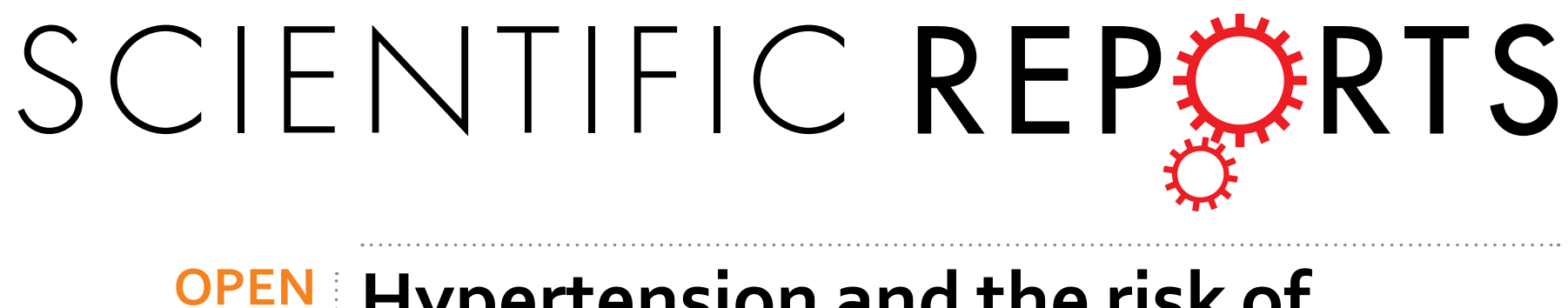

\title{
Hypertension and the risk of endometrial cancer: a systematic review and meta-analysis of case- control and cohort studies
}

Received: 27 October 2016

Accepted: 15 February 2017

Published: 07 April 2017

\author{
Dagfinn Aune ${ }^{1,2,3}$, Abhijit Sen $^{1} \&$ Lars J. Vatten ${ }^{1}$
}

A history of hypertension has been associated with increased risk of endometrial cancer in several studies, but the results have not been consistent. We conducted a systematic review and meta-analysis of case-control and cohort studies to clarify the association between hypertension and endometrial cancer risk. PubMed and Embase databases were searched up to $27^{\text {th }}$ of February 2016. Prospective and case-control studies which reported adjusted relative risk estimates and $95 \%$ confidence intervals of endometrial cancer associated with a hypertension diagnosis were included. Summary relative risks were estimated using a random effects model. Nineteen case-control studies and 6 cohort studies were included. The summary RR was $1.61\left(95 \% \mathrm{Cl}\right.$ : $\left.1.41-1.85, \mathrm{I}^{2}=86 \%\right)$ for all studies, $1.73(95 \% \mathrm{Cl}$ : $\left.1.45-2.06, \mathrm{I}^{2}=89 \%\right)$ for case-control studies and $1.32\left(95 \% \mathrm{Cl}: 1.12-1.56, \mathrm{I}^{2}=47 \%\right)$ for cohort studies. The association between hypertension and endometrial cancer was weaker, but still significant, among studies with adjustment for smoking, BMI, oral contraceptive use, and parity, compared to studies without such adjustment. This meta-analysis suggest an increased risk of endometrial cancer among patients with hypertension, however, further studies with more comprehensive adjustments for confounders are warranted to clarify the association.

Hypertension is a major cause of morbidity and mortality worldwide and is an established risk factor for coronary heart disease and stroke ${ }^{1,2}$. Globally a high systolic blood pressure accounted for 10.4 million deaths and 208.1 million disability-adjusted life-years (DALYs) in $2013^{3}$. Important risk factors for hypertension include overweight and obesity ${ }^{4}$, low physical activity ${ }^{5,6}$, high alcohol consumption ${ }^{7}$, dietary factors ${ }^{8-11}$, and use of non-narcotic analgesics ${ }^{12}$.

Endometrial cancer is the eighth most common type of cancer in women with approximately 320000 cases recorded in 2012, accounting for about $4.8 \%$ of all cancers in women $(2.3 \% \text { overall })^{13}$. It is more common in high-income countries than in low-income countries, however, its incidence has been increasing in populations undergoing urbanization and economic growth, in parallel with increasing obesity rates and sedentary lifestyles $^{14,15}$. Several risk factors for endometrial cancer have been established including excess body weight ${ }^{16}$, low physical activity ${ }^{17}$, diabetes history ${ }^{18}$, and use of unopposed hormone replacement therapy ${ }^{19}$. A history of hypertension has been evaluated as a risk factor for endometrial cancer in several case-control ${ }^{20-38}$ and cohort studies $^{39-44}$, and many ${ }^{20,21,24-26,28,30,32-39,42,44}$, but not all ${ }^{22,23,27,29,31,39,42,44}$ of these found an increased endometrial cancer risk. Because obesity and diabetes are important risk factors for both hypertension ${ }^{45,46}$ and endometrial cancer ${ }^{16,18}$ it is not clear whether the association between hypertension and endometrial cancer could be due to confounding by these factors because some studies did not adjust for BMI $\mathrm{BM}^{20,21,25,33,35,38}$ or diabetes ${ }^{20,21,24,25,28,29,33,35,38}$. We conducted a systematic review and meta-analysis of case-control and cohort studies that had investigated the association between hypertension and endometrial cancer risk with an aim of clarifying the strength of the association, possible sources of heterogeneity and potential confounding by other risk factors. Epidemiology and Biostatistics, Imperial College, London, UK. ${ }^{2}$ Department of Public Health and General Practice, Faculty of Medicine, Norwegian University of Science and Technology, Trondheim, Norway. ${ }^{3}$ Bjørknes University College, Oslo, Norway. Correspondence and requests for materials should be addressed to D.A. (email: d.aune@imperial.ac.uk) 


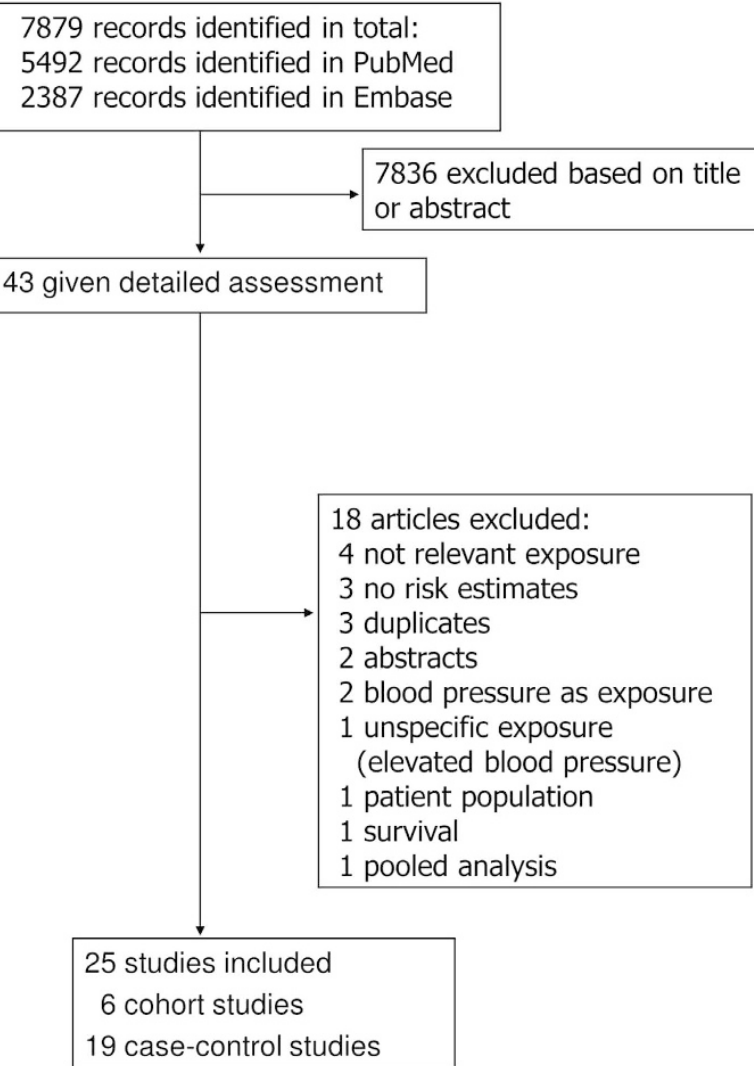

Figure 1. Flow-chart of study selection.

\section{Methods}

Search strategy and inclusion criteria. We searched the PubMed and Embase databases up to $27^{\text {th }}$ February 2016 for eligible studies. We used the following search terms in the PubMed search: (hypertension OR high blood pressure OR blood pressure OR risk factor) AND (endometrial cancer OR uterine cancer). We followed standard criteria for reporting meta-analyses ${ }^{47}$.

Study selection. We included published retrospective case-control studies and cohort studies that investigated the association between hypertension and the risk of endometrial cancer. Adjusted estimates of the relative risk (odds ratios and hazard ratios which were considered to be approximately equal given that endometrial cancer is a relatively uncommon cancer) had to be available with the $95 \%$ CIs in the publication. A list of excluded studies and exclusion reasons is provided in Supplementary Table 1. DA and AS conducted the study selection.

Data extraction. The following data were extracted from each study: The first author's last name, publication year, country where the study was conducted, study period, sample size, number of cases/controls, exposure and subgroups of tumor characteristics (low, moderate or high aggressiveness) or cancer type (type 1 vs. type 2), relative risks and $95 \%$ confidence intervals for the association and variables adjusted for in the analysis. Data were extracted by one reviewer (DA) and checked for accuracy by a second reviewer (AS).

Statistical methods. We calculated summary relative risks of developing endometrial cancer by history of hypertension using the random-effects model by DerSimonian and Laird ${ }^{48}$ which takes into account both within and between study variation (heterogeneity). The average of the natural logarithm of the relative risks was estimated and the relative risk from each study was weighted by the inverse of its variance ${ }^{49}$.

Heterogeneity between studies was evaluated using Q and $\mathrm{I}^{2}$ statistics ${ }^{50}$. Cochran's $\mathrm{Q}$ is calculated as the weighted sum of squared differences between individual study effects and the pooled effects across studies, with weights being those in the pooling method. $\mathrm{I}^{2}$ is a measure of how much of the heterogeneity that is due to between study variation rather than chance. $\mathrm{I}^{2}$-values of $25 \%, 50 \%$ and $75 \%$ indicates low, moderate and high heterogeneity respectively. We conducted main analyses (all studies combined) and stratified by study design (cohort studies, case-control studies) because of the greater potential for recall and selection bias in retrospective case-control studies and to investigate sources of potential heterogeneity. We also conducted subgroup analyses by other study characteristics such as sample size, number of cases, geographic location, and by adjustment for 
Hypertension and endometrial cancer

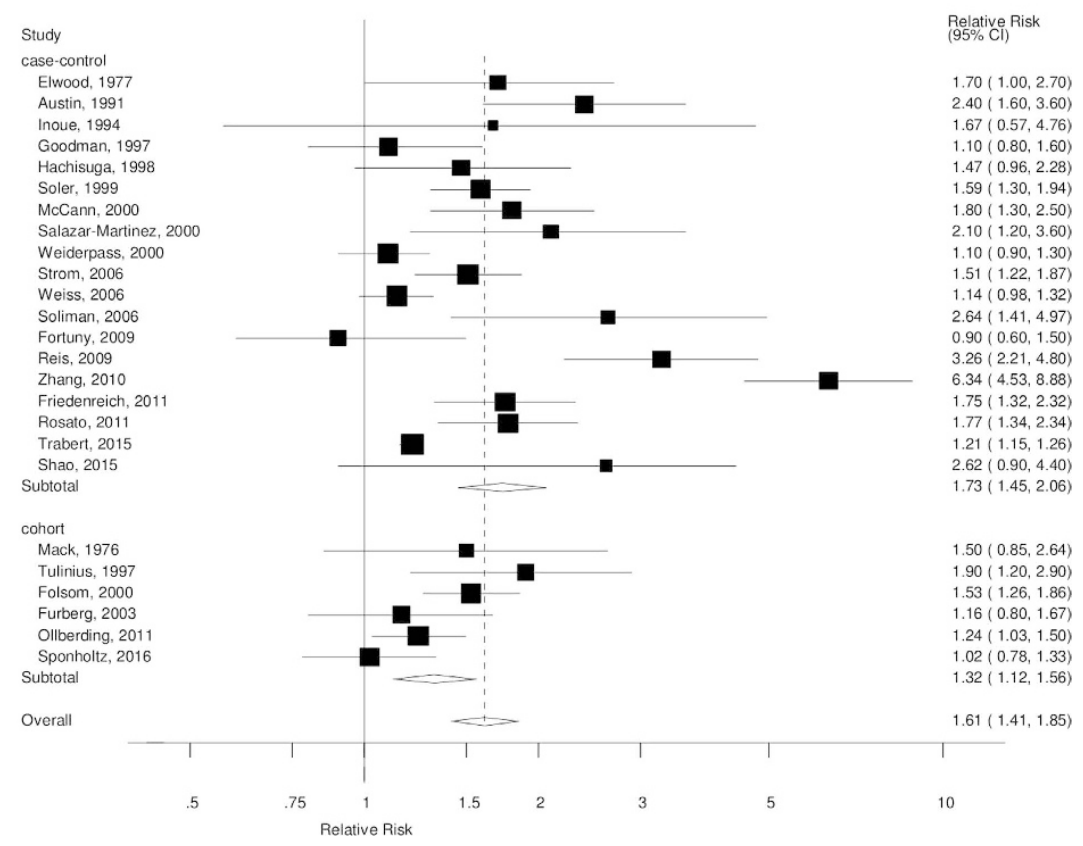

Figure 2. Hypertension and endometrial cancer, forest plot.

Funnel plot of hypertension and endometrial cancer

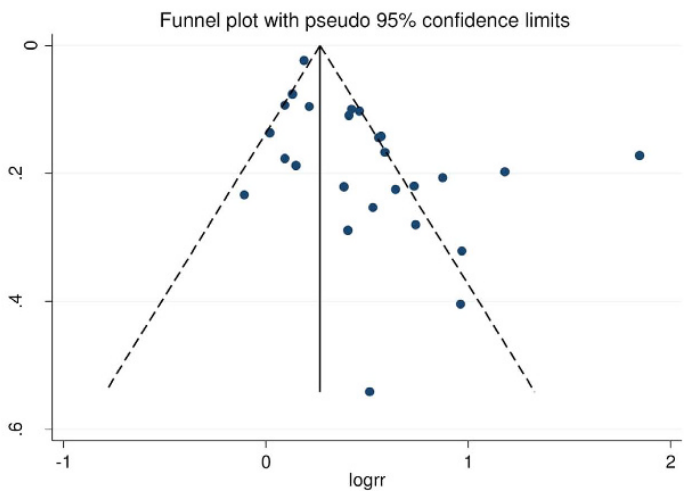

Figure 3. Hypertension and endometrial cancer, funnel plot.

confounding factors. We also conducted a stratified analysis by whether the articles explicitly stated that participants with prevalent hysterectomies at baseline were excluded, and/or whether participants with incident hysterectomies were censored during follow-up in cohort studies, or excluded from the control group in case-control studies.

Publication bias was assessed using Egger's test ${ }^{51}$ and Begg-Mazumdar's test ${ }^{52}$ and by inspection of funnel plots. Study quality was assessed using the Newcastle-Ottawa scale which ranks the studies on a scale from 0 to 9 based on the selection of the study population, comparability between cases and non-cases and the assessment of the outcome ${ }^{53}$. The statistical analyses were conducted using the software package Stata, version 13.0 software (StataCorp, Texas, US).

\section{Results}

Out of a total 7879 records identified by the search we included 25 studies with 28385 cases and 300598 participants in the meta-analysis of hypertension and endometrial cancer risk, including six cohort studies ${ }^{39-44}$ and nineteen case-control studies ${ }^{20-37}$ (Fig. 1 and Tables 1 and 2). Fourteen of the studies were from North-America, seven were from Europe, and four were from Asia (Tables 1 and 2). 


\begin{tabular}{|c|c|c|c|c|c|c|c|c|}
\hline $\begin{array}{l}\text { First author, } \\
\text { publication year, } \\
\text { country }\end{array}$ & $\begin{array}{l}\text { Number of } \\
\text { participants, age, } \\
\text { number of cases }\end{array}$ & Study period & $\begin{array}{l}\text { Assessment of } \\
\text { hypertension }\end{array}$ & $\begin{array}{c}\text { Cut-off for } \\
\text { hypertension }\end{array}$ & Exposure & Comparison & $\begin{array}{l}\text { Relative } \\
\text { risk }(95 \% \\
\text { confidence } \\
\text { interval) }\end{array}$ & $\begin{array}{l}\text { Adjustment for } \\
\text { confounders }\end{array}$ \\
\hline $\begin{array}{l}\text { Mack T et al., 1976, } \\
\text { USA }\end{array}$ & $\begin{array}{l}\text { Nested case-control } \\
\text { study: } 63 \text { cases } 252 \\
\text { controls }\end{array}$ & $\begin{array}{c}1971-1975, \sim 4 \\
\text { years follow-up }\end{array}$ & Self-reported & Not available & Hypertension & Yes vs. no & $\begin{array}{c}1.50 \\
(0.85-2.64)\end{array}$ & $\begin{array}{l}\text { Age, marital status, } \\
\text { community }\end{array}$ \\
\hline $\begin{array}{l}\text { Tulinius H et al., } \\
\text { 1997, Iceland }\end{array}$ & $\begin{array}{l}11580 \text { women, mean } \\
\text { age } 50.5 \text { years: } 98 \text { cases }\end{array}$ & $\begin{array}{c}1968-1995, \sim 15.1 \\
\text { years follow-up }\end{array}$ & Measured & Not available & Hypertension & Yes vs. no & $1.9(1.2-2.9)$ & Age \\
\hline $\begin{array}{l}\text { Folsom AR et al., } \\
2003, \text { USA }\end{array}$ & $\begin{array}{l}23335 \text { women, age } \\
\text { 55-69 years: } 415 \text { cases }\end{array}$ & $\begin{array}{l}\text { 1986-2000, } 15.7 \\
\text { years follow-up }\end{array}$ & Self-reported & Not available & Hypertension & Yes vs. no & $\begin{array}{c}1.53 \\
(1.26-1.86)\end{array}$ & Age \\
\hline \multirow{3}{*}{$\begin{array}{l}\text { Furberg AS et al., } \\
\text { 2003, Norway }\end{array}$} & \multirow{3}{*}{$\begin{array}{c}24460 \text { women, age } \\
20-49 \text { years: } 130 \text { cases }\end{array}$} & \multirow{3}{*}{$\begin{array}{l}\text { 1974-1981-1996, } \\
15.7 \text { years follow-up }\end{array}$} & \multirow{3}{*}{$\begin{array}{c}\text { Measured (mercury } \\
\text { sphygmo-manometer) }\end{array}$} & \multirow{3}{*}{$\underset{90 \mathrm{mmHg}}{\geq 140 / \geq}$} & \multirow{3}{*}{ Hypertension } & $\begin{array}{c}\text { Consistently } \\
\text { normotensive }\end{array}$ & 1.00 & \multirow{3}{*}{$\begin{array}{c}\text { Age, geographical region, } \\
\text { height, BMI, recreational } \\
\text { and occupational activity, } \\
\text { smoking, parity }\end{array}$} \\
\hline & & & & & & $\begin{array}{l}\text { Hypertensive in } \\
\text { one survey }\end{array}$ & $\begin{array}{c}1.11 \\
(0.70-1.77)\end{array}$ & \\
\hline & & & & & & $\begin{array}{l}\text { Consistently } \\
\text { hypertensive }\end{array}$ & $\begin{array}{c}1.24 \\
(0.69-2.25)\end{array}$ & \\
\hline $\begin{array}{l}\text { Ollberding NJ et al., } \\
\text { 2011, USA }\end{array}$ & $\begin{array}{l}46027 \text { postm. Women, } \\
\text { age } 45-75 \text { years: } 489 \\
\text { cases }\end{array}$ & $\begin{array}{l}\text { 1993/1996-2007, } \\
\text { 13.6 years follow-up }\end{array}$ & Self-reported & Not available & Hypertension & Yes vs. no & $\begin{array}{c}1.24 \\
(1.03-1.50)\end{array}$ & $\begin{array}{c}\text { Age, race/ethnicity, age at } \\
\text { cohort entry, total calories, } \\
\text { BMI, age at menarche, } \\
\text { age at menopause, parity, } \\
\text { duration of OC use, HRT } \\
\text { use, smoking status, } \\
\text { diabetes }\end{array}$ \\
\hline $\begin{array}{l}\text { Sponholtz TR et al., } \\
\text { 2016, USA }\end{array}$ & $\begin{array}{c}47577 \text { women, age } \\
\text { 21-69 years: } 274 \text { cases }\end{array}$ & $\begin{array}{c}\text { 1995-2013, } 14 \text { years } \\
\text { follow-up }\end{array}$ & Self-reported & Not available & Hypertension & Yes vs. no & $\begin{array}{c}1.02 \\
(0.78-1.33)\end{array}$ & $\begin{array}{l}\text { Age, study period, age } \\
\text { at menarche, parity, } \\
\text { menopausal status, } \\
\text { OC use, estrogen-only } \\
\text { hormone use, estrogen } \\
\text { plus progestin hormone } \\
\text { use, smoking status, BMI, } \\
\text { vigorous physical activity, } \\
\text { statin use, metformin use }\end{array}$ \\
\hline
\end{tabular}

Table 1. Prospective studies of hypertension and endometrial cancer. BMI = body mass index, OC use $=$ oral contraceptive use, HRT use $=$ hormone replacement therapy use.

The summary RR for all studies was 1.61 (95\% CI: $\left.1.41-1.85, \mathrm{I}^{2}=86 \%\right)$, and it was 1.73 (95\% CI: $1.45-2.06$, $\left.\mathrm{I}^{2}=89 \%\right)$ for case-control studies and 1.32 (95\% CI: $\left.1.12-1.56, \mathrm{I}^{2}=47 \%\right)$ for cohort studies (Fig. 2), however, the test for heterogeneity by study design was not significant, $\mathrm{p}=0.19$. In sensitivity analyses excluding one study at a time the summary RR ranged from 1.49 (95\% CI: 1.34-1.65) when excluding the study by Zhang et al. ${ }^{33}$ to 1.65 (95\% CI: 1.41-1.94) when excluding the study by Trabert et al. ${ }^{36}$. There was evidence of publication bias with Egger's test, $\mathrm{p}=0.005$ (Fig. 3), however, when stratified by study design this was observed among case-control studies, $\mathrm{p}=0.007$, but not among cohort studies, $\mathrm{p}=0.78$

Subgroup and sensitivity analyses, study quality assessment. There were positive associations in almost all subgroup analyses (Table 3), and although there was no heterogeneity when stratified by study design, geographic location or number of cases, there was indication of heterogeneity when studies were stratified by confounding factors including smoking $(\mathrm{p}=0.02)$, BMI $(\mathrm{p}=0.003)$, oral contraceptive use $(\mathrm{p}=0.02)$, hormone replacement therapy $(\mathrm{p}=0.08)$, parity $(\mathrm{p}=0.03)$, and age at menopause $(\mathrm{p}=0.07)$, with weaker, but still significant associations among studies with such adjustments. When we conducted sensitivity analyses removing one study at a time, the size of the summary estimate persisted and did not vary substantially (Supplementary Table 2).

In a further sensitivity analysis we also conducted a subgroup analysis by whether the studies explicitly stated that they excluded participants with prevalent hysterectomies at baseline and/or stated that they censored participants at the time of incident hysterectomy (cohort studies) or excluded participants who had undergone hysterectomy from the control group (case-control studies). The summary RR was 1.51 (95\% CI: 1.28-1.78, $\mathrm{I}^{2}=88.5 \%$ ) for studies with such exclusions or censoring and 1.81 (95\% CI: 1.49-2.20, $\mathrm{I}^{2}=56.5 \%$ ) for studies without such exclusions or censoring.

In a sensitivity analysis we also included a pooled analysis which assessed the association between quintiles of systolic blood pressure and endometrial cancer risk ${ }^{54}$, using the relative risk for the highest vs. the lowest quintile of systolic blood pressure. The results were not materially altered, summary RR $=1.61$ (95\% CI: $1.42-1.83$, $\left.\mathrm{I}^{2}=38 \%\right)$ for all studies and 1.33 (95\% CI: $\left.1.16-1.52, \mathrm{I}^{2}=86 \%\right)$ for cohort studies. Further including another cohort study ${ }^{55}$ which reported on elevated blood pressure $(\geq 130 / \geq 85 \mathrm{vs.}<130 /<85 \mathrm{~mm} / \mathrm{Hg}$ ) or self-reported hypertension, not only hypertension, did also not substantially alter the results, summary RR $=1.57$ (95\% CI: $\left.1.38-1.78, \mathrm{I}^{2}=85 \%\right)$ for all studies and summary $\mathrm{RR}=1.28$ (95\% CI: $\left.1.12-1.48, \mathrm{I}^{2}=46 \%\right)$ for cohort studies. Mean (median) study quality scores were 7.3 (7.0) for all studies combined, 7.3 (7.0) for case-control studies, and 7.3 (7.0) for cohort studies).

\section{Discussion}

To our knowledge this is the first meta-analysis of published observational studies of hypertension and the risk of endometrial cancer and our results confirm that hypertension is a strong risk factor for endometrial cancer 


\begin{tabular}{|c|c|c|c|c|c|c|c|c|}
\hline $\begin{array}{l}\text { First author, publication } \\
\text { year, country }\end{array}$ & $\begin{array}{l}\text { Number of } \\
\text { cases and } \\
\text { controls, age }\end{array}$ & $\begin{array}{c}\text { Study } \\
\text { period }\end{array}$ & $\begin{array}{l}\text { Assessment of } \\
\text { hypertension }\end{array}$ & $\begin{array}{c}\text { Cut-off for } \\
\text { hypertension }\end{array}$ & $\begin{array}{l}\text { Exposure, } \\
\text { subgroup, } \\
\text { outcome }\end{array}$ & Comparison & $\begin{array}{l}\text { Relative } \\
\text { risk }(95 \% \\
\text { confidence } \\
\text { interval })\end{array}$ & $\begin{array}{l}\text { Adjustment for confounders or } \\
\text { matching variables }\end{array}$ \\
\hline $\begin{array}{l}\text { Elwood JM et al., 1977, } \\
\text { USA }\end{array}$ & $\begin{array}{l}212 \text { cases } 1198 \\
\text { population } \\
\text { controls Age } \\
55-69 \text { years }\end{array}$ & $1965-1969$ & Self-reported & Not available & Hypertension & Yes vs. no & $1.7(1.0-2.7)$ & Year of birth \\
\hline $\begin{array}{l}\text { Austin H et al., 1991, } \\
\text { USA }\end{array}$ & \begin{tabular}{|c|}
168 cases 334 \\
hospital controls \\
Age $40-82$ years
\end{tabular} & $1985-1988$ & $\begin{array}{l}\text { Self-reported } \\
\text { (interview) }\end{array}$ & Not available & Hypertension & Yes vs. no & $2.4(1.6-3.6)$ & Age, race, years of schooling \\
\hline $\begin{array}{l}\text { Inoue M et al., 1994, } \\
\text { Japan }\end{array}$ & \begin{tabular}{|c|}
143 cases 143 \\
hospital controls \\
Age $22-79$ years
\end{tabular} & $1979-1992$ & $\begin{array}{l}\text { Medical } \\
\text { records }\end{array}$ & Not available & Hypertension & Yes vs. no & $1.67(0.57-4.76)$ & $\begin{array}{l}\text { Age, obesity, personal cancer } \\
\text { history, diabetes mellitus, parity }\end{array}$ \\
\hline $\begin{array}{l}\text { Goodman MT et al., } \\
\text { 1997, USA }\end{array}$ & $\begin{array}{l}332 \text { cases } 511 \\
\text { population } \\
\text { controls Age } \\
18-84 \text { years }\end{array}$ & $1985-1993$ & $\begin{array}{l}\text { Self-reported } \\
\text { (interview }\end{array}$ & Not available & Hypertension & Yes vs. no & $1.1(0.8-1.6)$ & $\begin{array}{l}\text { Age, ethnicity, pregnancy history, } \\
\text { OC use, unopposed estrogen use, } \\
\text { diabetes history, BMI }\end{array}$ \\
\hline $\begin{array}{l}\text { Hachisuga T et al., 1998, } \\
\text { Japan }\end{array}$ & $\begin{array}{c}242 \text { cases } 1021 \\
\text { hospital controls } \\
\text { Age } 20-79 \text { years }\end{array}$ & $1980-1989$ & $\begin{array}{l}\text { Medical } \\
\text { records }\end{array}$ & Not available & Hypertension & Yes vs. no & $1.47(0.96-2.28)$ & $\begin{array}{l}\text { Age, parity, BMI, diabetes } \\
\text { mellitus }\end{array}$ \\
\hline Soler M et al., 1999, Italy & $\begin{array}{c}745 \text { cases } 3054 \\
\text { hospital controls } \\
\text { Age }<75 \text { years }\end{array}$ & $1983-1996$ & $\begin{array}{l}\text { Self-reported } \\
\text { (interview) }\end{array}$ & Not available & $\begin{array}{c}\text { Treated } \\
\text { hypertension }\end{array}$ & Yes vs. no & $1.59(1.30-1.94)$ & $\begin{array}{l}\text { Age, area of residence, education, } \\
\text { smoking, alcohol, parity, } \\
\text { menopausal status, BMI }\end{array}$ \\
\hline $\begin{array}{l}\text { McCann SE et al., 2000, } \\
\text { USA }\end{array}$ & $\begin{array}{l}232 \text { cases } 639 \\
\text { population } \\
\text { controls Age } \\
40-85 \text { years }\end{array}$ & $1986-1991$ & $\begin{array}{l}\text { Self-reported } \\
\text { (interview) }\end{array}$ & Not available & Hypertension & Yes vs. no & $1.8(1.3-2.5)$ & Age \\
\hline $\begin{array}{l}\text { Salazar-Martinez E et al., } \\
\text { 2000, Mexico }\end{array}$ & $\begin{array}{c}85 \text { cases } 668 \\
\text { population } \\
\text { controls Mean } \\
\text { age } 61.7 / 60.2 \\
\text { years }\end{array}$ & 1995-1997 & $\begin{array}{l}\text { Self-reported } \\
\text { (interview) }\end{array}$ & Not available & Hypertension & Yes vs. no & $2.1(1.2-3.6)$ & $\begin{array}{l}\text { Age, anovulatory index, smoking, } \\
\text { physical activity, menopausal } \\
\text { status, diabetes, BMI }\end{array}$ \\
\hline $\begin{array}{l}\text { Weiderpass E et al., 2000, } \\
\text { Sweden }\end{array}$ & $\begin{array}{c}709 \text { cases } 3368 \\
\text { population } \\
\text { controls Age } \\
50-74 \text { years } \\
\end{array}$ & 1994-1995 & Self-reported & Not available & Hypertension & Yes vs. no & $1.1(0.9-1.3)$ & $\begin{array}{l}\text { Age, age at menarche, parity, age } \\
\text { at last birth, age at menopause, } \\
\text { smoking, OC use, HRT, diabetes } \\
\text { mellitus, recent BMI }\end{array}$ \\
\hline $\begin{array}{l}\text { Strom BL et al., 2006, } \\
\text { USA }\end{array}$ & $\begin{array}{l}511 \text { cases } 1412 \\
\text { population } \\
\text { controls Age } \\
50-79 \text { years }\end{array}$ & 1999-2002 & $\begin{array}{l}\text { Self-reported } \\
\text { (interview) }\end{array}$ & Not available & Hypertension & Yes vs. no & $1.51(1.22-1.87)$ & $\begin{array}{l}\text { Age, ethnicity, education, BMI, } \\
\text { number of full-term pregnancies, } \\
\text { years of menses, type of } \\
\text { menopause, smoking status, years } \\
\text { of smoking, OC use }\end{array}$ \\
\hline \multirow{3}{*}{$\begin{array}{l}\text { Weiss JM et al., 2006, } \\
\text { USA }\end{array}$} & \multirow{3}{*}{$\begin{array}{l}1304 \text { cases } 1779 \\
\text { population } \\
\text { controls Age } \\
45-74 \text { years }\end{array}$} & 1985-1991 & \multirow{3}{*}{$\begin{array}{l}\text { Self-reported } \\
\text { (interview) }\end{array}$} & \multirow{3}{*}{ Not available } & $\begin{array}{l}\text { Hypertension, } \\
\text { low tumor } \\
\text { aggressiveness }\end{array}$ & Yes vs. no & $1.2(1.0-1.6)$ & \multirow{3}{*}{$\begin{array}{l}\text { Age, } \mathrm{HRT}, \mathrm{BMI} \text {, county of } \\
\text { residence, referent year }\end{array}$} \\
\hline & & 1994-1995 & & & $\begin{array}{c}\text { Hypertension, } \\
\text { moderate tumor } \\
\text { aggressiveness }\end{array}$ & Yes vs. no & $1.1(0.9-1.4)$ & \\
\hline & & $1997-1999$ & & & $\begin{array}{l}\text { Hypertension, } \\
\text { high tumor } \\
\text { aggressiveness }\end{array}$ & Yes vs. no & $1.1(0.7-1.6)$ & \\
\hline $\begin{array}{l}\text { Soliman PT et al., 2006, } \\
\text { USA }\end{array}$ & \begin{tabular}{|c|}
117 cases 238 \\
hospital controls \\
Age $25-88$ years
\end{tabular} & 2000-2004 & $\begin{array}{l}\text { Medical } \\
\text { records }\end{array}$ & Not available & Hypertension & Yes vs. No & $2.64(1.41-4.97)$ & Age, BMI, diabetes \\
\hline $\begin{array}{l}\text { Fortuny J et al., 2009, } \\
\text { USA }\end{array}$ & $\begin{array}{c}469 \text { cases } 467 \\
\text { population } \\
\text { controls Age } \geq \\
21 \text { years }\end{array}$ & 2001-2005 & $\begin{array}{l}\text { Self-reported } \\
\text { (interview) }\end{array}$ & Not available & Hypertension & Yes vs. no & $0.9(0.6-1.5)$ & $\begin{array}{l}\text { Age, BMI, education, race, age at } \\
\text { menarche, HRT, OC use, age at } \\
\text { menopause, parity, smoking, FH } \\
\text { - EC, type } 2 \text { diabetes, biguanides, } \\
\text { insulin, sulphonylureas, } \\
\text { hypercholesterolemia, statins, } \\
\text { fibrates, ACE-inhibitors, } \\
\text { beta-blockers, calcium channel } \\
\text { blockers, angiotensin 2 } \\
\text { receptor antagonists, thiazide } \\
\text { diuretics, loop diuretics, } \mathrm{K} \\
\text { sparing diuretics, osteoporosis, } \\
\text { biphosphonates, calcitonin, } \\
\text { endometrial cancer fibroids }\end{array}$ \\
\hline $\begin{array}{l}\text { Reis N et al., 2009, } \\
\text { Turkey }\end{array}$ & $\begin{array}{c}285 \text { cases } 1050 \\
\text { hospital controls } \\
\text { Age } 43-76 \text { years }\end{array}$ & $2002-2003$ & $\begin{array}{l}\text { Self-report } \\
\text { of treated } \\
\text { hypertension } \\
\text { or physician- } \\
\text { diagnosis } \\
\text { (interview) }\end{array}$ & Not available & Hypertension & Yes vs. no & $3.26(2.21-4.80)$ & $\begin{array}{l}\text { Age, education, diabetes, parity, } \\
\text { age at menarche, HRT use, 1st } \\
\text { degree relative history of breast, } \\
\text { endometrial cancer or colorectal } \\
\text { cancer, 2nd degree relative with } \\
\text { history of breast and ovarian } \\
\text { cancer }\end{array}$ \\
\hline
\end{tabular}




\begin{tabular}{|c|c|c|c|c|c|c|c|c|}
\hline $\begin{array}{l}\text { First author, publication } \\
\text { year, country }\end{array}$ & $\begin{array}{l}\text { Number of } \\
\text { cases and } \\
\text { controls, age }\end{array}$ & $\begin{array}{l}\text { Study } \\
\text { period }\end{array}$ & $\begin{array}{l}\text { Assessment of } \\
\text { hypertension }\end{array}$ & $\begin{array}{c}\text { Cut-off for } \\
\text { hypertension }\end{array}$ & $\begin{array}{l}\text { Exposure, } \\
\text { subgroup, } \\
\text { outcome }\end{array}$ & Comparison & $\begin{array}{l}\text { Relative } \\
\text { risk }(95 \% \\
\text { confidence } \\
\text { interval) }\end{array}$ & $\begin{array}{l}\text { Adjustment for confounders or } \\
\text { matching variables }\end{array}$ \\
\hline \multirow{3}{*}{$\begin{array}{l}\text { Zhang Y et al., 2010, } \\
\text { China }\end{array}$} & \multirow{3}{*}{$\begin{array}{c}942 \text { cases } 1721 \\
\text { healthy hospital } \\
\text { controls Age NA }\end{array}$} & \multirow{3}{*}{$2004-2008$} & \multirow{3}{*}{ Medical record } & \multirow{3}{*}{$\begin{array}{l}\geq 140 / \geq \\
90 \mathrm{~mm} / \mathrm{Hg}\end{array}$} & $\begin{array}{l}\text { Hypertension, } \\
\text { all }\end{array}$ & Yes vs. no & $6.34(4.53-8.88)$ & \multirow{3}{*}{ Age } \\
\hline & & & & & $\begin{array}{c}\text { Hypertension, } \\
\text { type } 1 \\
\text { endometrial } \\
\text { cancer }\end{array}$ & Yes vs. no & $6.39(4.50-9.06)$ & \\
\hline & & & & & $\begin{array}{c}\text { Hypertension, } \\
\text { type } 2 \\
\text { endometrial } \\
\text { cancer }\end{array}$ & Yes vs. no & $\begin{array}{l}6.63(4.01- \\
10.94)\end{array}$ & \\
\hline $\begin{array}{l}\text { Friedenreich CM et al., } \\
\text { 2011, Canada }\end{array}$ & $\begin{array}{l}515 \text { cases } 962 \\
\text { population } \\
\text { controls Age } \\
30-79 \text { years }\end{array}$ & $2002-2006$ & $\begin{array}{l}\text { Self-reported } \\
\text { (interview) }\end{array}$ & Not available & $\begin{array}{l}\text { Ever diagnosed } \\
\text { and treated for } \\
\text { hypertension }\end{array}$ & Yes vs. no & $1.75(1.32-2.32)$ & $\begin{array}{c}\text { Age, age }{ }^{2} \text {, age at menarche, } \\
\text { number of pregnancies } \geq 20 \\
\text { weeks gestation, type of HRT, } \\
\text { waist circumference, triglycerides, } \\
\text { HDL-cholesterol, fasting blood } \\
\text { glucose }\end{array}$ \\
\hline $\begin{array}{l}\text { Rosato V et al., 2011, } \\
\text { Italy }\end{array}$ & \begin{tabular}{|c|}
454 cases 798 \\
hospital controls \\
Age $18-79 / 19-$ \\
79 years \\
\end{tabular} & $1992-2006$ & $\begin{array}{l}\text { Self-reported } \\
\text { (interview) }\end{array}$ & Not available & Hypertension & Yes vs. no & $1.77(1.34-2.34)$ & $\begin{array}{l}\text { Age, study center, year of } \\
\text { interview, education, age at } \\
\text { menarche, parity, menopausal } \\
\text { status, OC use, HRT use }\end{array}$ \\
\hline $\begin{array}{l}\text { Trabert B et al., 2015, } \\
\text { USA }\end{array}$ & $\begin{array}{c}19323 \text { cases } \\
100751 \\
\text { population } \\
\text { controls Age } \geq \\
65 \text { years }\end{array}$ & $1993-2007$ & $\begin{array}{l}\text { Medical } \\
\text { records }\end{array}$ & Not available & Hypertension & Yes vs. no & $1.21(1.15-1.26)$ & $\begin{array}{c}\text { Age, diagnosis date, race/ } \\
\text { ethnicity, registry area, tobacco } \\
\text { use, overweight/obesity, impaired } \\
\text { fasting glucose, high triglycerides }\end{array}$ \\
\hline Shao Y et al., 2015, China & $\begin{array}{c}128 \text { cases } 294 \\
\text { hospital controls } \\
\text { Age } 22-43 \text { years }\end{array}$ & 2010-2013 & $\begin{array}{l}\text { Self-reported } \\
\text { (interview) }\end{array}$ & Not available & Hypertension & Yes vs. no & $2.62(0.90-4.40)$ & $\begin{array}{c}\text { Age, time of day of blood } \\
\text { collection, CRP, IL-6, TNF- } \alpha \\
\text {, insulin, C-peptide, SHBG, } \\
\text { birth weight }>4 \text { kg, BMI, WHR, } \\
\text { diabetes, age at menarche, FH } \\
\text { - cancer }\end{array}$ \\
\hline
\end{tabular}

Table 2. Case-control studies of hypertension and endometrial cancer. ACE-inhibitor = angiotensinconverting enzyme inhibitor, $\mathrm{BMI}=$ body mass index, $\mathrm{CRP}=\mathrm{C}$-reactive protein, $\mathrm{FH}-\mathrm{EC}=$ family history of endometrial cancer, HDL-cholesterol = high-density lipoprotein cholesterol, HRT use = hormone replacement therapy use, IL- $6=$ interleukin- 6 , NA $=$ not available, OC use $=$ oral contraceptive use, TNF- $\alpha=$ tumor necrosis factor $\alpha, \mathrm{WHR}=$ waist-to-hip ratio.

with a $61 \%$ increase in the relative risk, however, the association was weaker in cohort studies $(\mathrm{RR}=1.32)$ than among case-control studies $(R R=1.73)$. These findings are consistent with a large cohort study of 290000 women in Austria, Norway and Sweden which found an increased endometrial cancer risk with increasing levels of diastolic blood pressure and in particular, systolic blood pressure ${ }^{54}$. The results also persisted in a sensitivity analysis including the results from this cohort study ${ }^{54}$ as well as the EPIC study ${ }^{55}$, which reported on elevated blood pressure or hypertension.

The present meta-analysis has some limitations. As hypertension is a condition that is strongly related to lifestyle factors and some medical conditions including diet, BMI, physical activity, and diabetes we cannot entirely exclude the possibility that the observed association between hypertension and endometrial cancer risk at least partly could be due to confounding. We found that the association was weaker, but still statistically significant, among studies that adjusted for smoking, BMI, oral contraceptive use, hormone replacement use, parity and age at menopause ( $\mathrm{RR}=1.14-1.34$ for studies with such adjustment vs. $1.74-2.10$ for studies without such adjustment). However, because there was still a significant association in subgroups that adjusted for these factors it could indicate that there is an adverse effect of hypertension on endometrial cancer risk, but that it may be slightly weaker than what was suggested from the overall summary estimates. Because the original studies did not stratify for BMI or diabetes it was not possible for us to investigate whether the association was limited to specific weight classes or if it was modified by diabetes status.

We also found that the positive association between hypertension and endometrial cancer persisted when the studies were stratified by whether participants with prevalent hysterectomies at baseline were excluded and/ or whether participants with incident hysterectomies were censored, or whether prevalent hysterectomies were excluded from the control group. Hypertension may also be related to hysterectomies ${ }^{56-58}$, and could potentially bias the risk estimates, however, any bias would most likely be toward the null. We cannot exclude the possibility of residual confounding from other risk factors such as use of intrauterine device ${ }^{59}$, polycystic ovarial syndrome $e^{60}$, or other potential risk factors that the original studies may not have adjusted for.

Case-control studies are more likely to be affected by certain biases, such as recall bias and selection bias. Because we included both case-control and cohort studies there is a possibility that recall or selection bias might have affected the results in the case-control studies and the overall summary estimate. Although the association appeared to be stronger in case-control studies than among cohort studies, there was still a significant association among cohort studies, which suggest that recall bias or selection biases does not entirely explain the observed association. In addition, there was some indication of publication bias with Egger's test, but this appeared to 


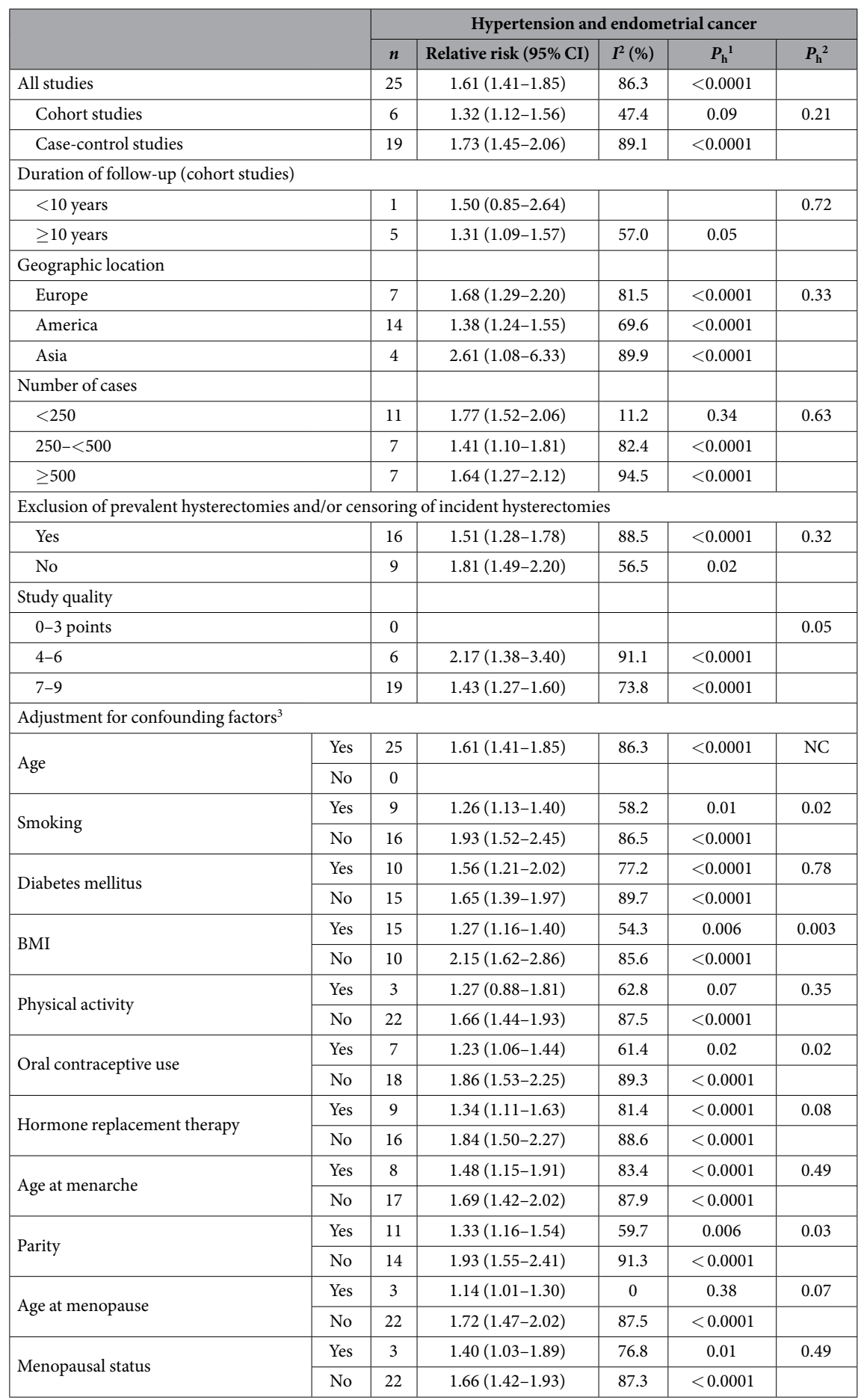

Table 3. Subgroup analyses of hypertension and endometrial cancer. $n$ denotes the number of studies, ${ }^{1} \mathrm{P}$ for heterogeneity within each subgroup, ${ }^{2} \mathrm{P}$ for heterogeneity between subgroups with meta-regression analysis. $\mathrm{NC}$, not calculable because no studies were present in one of the subgroups.

be restricted to the analyses of case-control studies and all studies combined, and was not observed among the cohort studies.

The biological mechanism(s) that may explain an adverse effect of hypertension on endometrial cancer risk are unclear at present. It has been suggested that long-term hypertension may lead to cellular senescence and inhibition of apoptosis ${ }^{61}$. It has also been suggested that medications used for the treatment of hypertension could increase cancer risk, however, a meta-analysis found little evidence of an association with overall cancer ${ }^{62}$, and a cohort study found no relation with female genital cancers ${ }^{63}$, although few studies have specifically investigated endometrial cancer. 
Strengths of the present meta-analysis include the comprehensive search strategy, the detailed subgroup and sensitivity analyses, and the large sample size providing a more robust estimate of the association between hypertension and endometrial cancer risk. To date relatively few studies have investigated the association between hypertensive disorders of pregnancy and endometrial cancer risk with one study suggesting an increased risk with hypertensive disorders overall ${ }^{64}$, while another study found no association with preeclampsia overall, although an increased risk was observed with early-onset preeclampsia ${ }^{65}$. Any further studies could better assess the causality of the observed association between hypertension and endometrial cancer by using genetic risk scores for hypertension $^{66,67}$. In addition, clarification of potential effect modification by age at exposure, BMI and diabetes status, and further studies of the association with subtypes of endometrial cancer are needed.

In conclusion, the results from this systematic review and meta-analysis suggest that women with hypertension may have a $61 \%$ increase in the relative risk of developing endometrial cancer. Any further studies should clarify potential effect modification by age, BMI and diabetes status, and the causality of the observed association, as well as the potential underlying mechanism(s).

\section{References}

1. Lewington, S., Clarke, R., Qizilbash, N., Peto, R. \& Collins, R. Age-specific relevance of usual blood pressure to vascular mortality: a meta-analysis of individual data for one million adults in 61 prospective studies. Lancet 360, 1903-1913 (2002).

2. Lewington, S. et al. The Burden of Hypertension and Associated Risk for Cardiovascular Mortality in China. JAMA Intern. Med. 176, 524-532 (2016).

3. GBD 2013 Mortality and Causes of Death Collaborators. Global, regional, and national age-sex specific all-cause and cause-specific mortality for 240 causes of death, 1990-2013: a systematic analysis for the Global Burden of Disease Study 2013. Lancet 385, 117-171 (2015).

4. Field, A. E. et al. Impact of overweight on the risk of developing common chronic diseases during a 10-year period. Arch. Intern. Med. 161, 1581-1586 (2001).

5. Hernelahti, M., Kujala, U. \& Kaprio, J. Stability and change of volume and intensity of physical activity as predictors of hypertension. Scand. J. Public Health 32, 303-309 (2004).

6. Williams, P. T. A cohort study of incident hypertension in relation to changes in vigorous physical activity in men and women. J. Hypertens. 26, 1085-1093 (2008).

7. Okubo, Y. et al. Association of alcohol consumption with incident hypertension among middle-aged and older Japanese population: the Ibarakai Prefectural Health Study (IPHS). Hypertension 63, 41-47 (2014).

8. Wang, L., Manson, J. E., Buring, J. E. \& Sesso, H. D. Meat intake and the risk of hypertension in middle-aged and older women. J. Hypertens. 26, 215-222 (2008).

9. Wang, L., Manson, J. E., Gaziano, J. M., Buring, J. E. \& Sesso, H. D. Fruit and vegetable intake and the risk of hypertension in middleaged and older women. Am. J. Hypertens. 25, 180-189 (2012).

10. Wang, L. et al. Whole- and refined-grain intakes and the risk of hypertension in women. Am. J. Clin. Nutr. 86, 472-479 (2007).

11. He, F. J., Li, J. \& MacGregor, G. A. Effect of longer-term modest salt reduction on blood pressure. Cochrane Database Syst. Rev. 4, CD004937 (2013)

12. Forman, J. P., Stampfer, M. J. \& Curhan, G. C. Non-narcotic analgesic dose and risk of incident hypertension in US women. Hypertension 46, 500-507 (2005).

13. Torre, L. A. et al. Global cancer statistics, 2012. CA Cancer J. Clin. 65, 87-108 (2015).

14. Matsuda, T. et al. Cancer incidence and incidence rates in Japan in 2005: based on data from 12 population-based cancer registries in the Monitoring of Cancer Incidence in Japan (MCIJ) project. Jpn. J Clin Oncol. 41, 139-147 (2011).

15. Huang, C. Y. et al. Nationwide surveillance in uterine cancer: survival analysis and the importance of birth cohort: 30 -year population-based registry in Taiwan. PLoS One 7, e51372 (2012).

16. Aune, D. et al. Anthropometric factors and endometrial cancer risk: a systematic review and dose-response meta-analysis of prospective studies. Ann. Oncol. 26, 1635-1648 (2015).

17. Cust, A. E., Armstrong, B. K., Friedenreich, C. M., Slimani, N. \& Bauman, A. Physical activity and endometrial cancer risk: a review of the current evidence, biologic mechanisms and the quality of physical activity assessment methods. Cancer Causes Control 18, 243-258 (2007).

18. Friberg, E., Orsini, N., Mantzoros, C. S. \& Wolk, A. Diabetes mellitus and risk of endometrial cancer: a meta-analysis. Diabetologia (2007).

19. Bernstein, L. The risk of breast, endometrial and ovarian cancer in users of hormonal preparations. Basic Clin. Pharmacol. Toxicol. 98, 288-296 (2006)

20. Elwood, J. M., Cole, P., Rothman, K. J. \& Kaplan, S. D. Epidemiology of endometrial cancer. J. Natl. Cancer Inst. 59, 1055-1060 (1977).

21. Austin, H., Austin, J. M. Jr., Partridge, E. E., Hatch, K. D. \& Shingleton, H. M. Endometrial cancer, obesity, and body fat distribution. Cancer Res. 51, 568-572 (1991).

22. Inoue, M. et al. A case-control study on risk factors for uterine endometrial cancer in Japan. Jpn. J. Cancer Res. 85, 346-350 (1994).

23. Goodman, M. T. et al. Diet, body size, physical activity, and the risk of endometrial cancer. Cancer Res. 57, 5077-5085 (1997).

24. Soler, M. et al. Hypertension and hormone-related neoplasms in women. Hypertension 34, 320-325 (1999).

25. McCann, S. E. et al. Diet in the epidemiology of endometrial cancer in western New York (United States). Cancer Causes Control 11, 965-974 (2000)

26. Salazar-Martinez, E. et al. Case-control study of diabetes, obesity, physical activity and risk of endometrial cancer among Mexican women. Cancer Causes Control 11, 707-711 (2000).

27. Weiderpass, E. et al. Body size in different periods of life, diabetes mellitus, hypertension, and risk of postmenopausal endometrial cancer (Sweden). Cancer Causes Control 11, 185-192 (2000).

28. Strom, B. L. et al. Case-control study of postmenopausal hormone replacement therapy and endometrial cancer. Am. J. Epidemiol. 164, 775-786 (2006).

29. Weiss, J. M. et al. Risk factors for the incidence of endometrial cancer according to the aggressiveness of disease. Am. J. Epidemiol. 164, 56-62 (2006).

30. Soliman, P. T. et al. Association between adiponectin, insulin resistance, and endometrial cancer. Cancer 106, 2376-2381 (2006).

31. Fortuny, J. et al. Risk of endometrial cancer in relation to medical conditions and medication use. Cancer Epidemiol. Biomarkers Prev. 18, 1448-1456 (2009).

32. Reis, N. \& Beji, N. K. Risk factors for endometrial cancer in Turkish women: results from a hospital-based case-control study. Eur. J. Oncol. Nurs. 13, 122-127 (2009).

33. Zhang, Y. et al. The association between metabolic abnormality and endometrial cancer: a large case-control study in China. Gynecol. Oncol. 117, 41-46 (2010). 
34. Friedenreich, C. M. et al. Case-control study of the metabolic syndrome and metabolic risk factors for endometrial cancer. Cancer Epidemiol. Biomarkers Prev. 20, 2384-2395 (2011).

35. Rosato, V. et al. Metabolic syndrome and endometrial cancer risk. Ann. Oncol. 22, 884-889 (2011).

36. Trabert, B. et al. Metabolic syndrome and risk of endometrial cancer in the united states: a study in the SEER-medicare linked database. Cancer Epidemiol. Biomarkers Prev. 24, 261-267 (2015).

37. Shao, Y. et al. Insulin is an important risk factor of endometrial cancer among premenopausal women: a case-control study in China. Tumour. Biol. (2015).

38. La Vecchia, C., Decarli, A., Fasoli, M. \& Gentile, A. Nutrition and diet in the etiology of endometrial cancer. Cancer 57, 1248-1253 (1986).

39. Mack, T. M. et al. Estrogens and endometrial cancer in a retirement community. N. Engl. J Med. 294, 1262-1267 (1976).

40. Tulinius, H., Sigfusson, N., Sigvaldason, H., Bjarnadottir, K. \& Tryggvadottir, L. Risk factors for malignant diseases: a cohort study on a population of 22,946 Icelanders. Cancer Epidemiol. Biomarkers Prev. 6, 863-873 (1997).

41. Folsom, A. R., Demissie, Z. \& Harnack, L. Glycemic index, glycemic load, and incidence of endometrial cancer: the Iowa women's health study. Nutr. Cancer 46, 119-124 (2003).

42. Furberg, A. S. \& Thune, I. Metabolic abnormalities (hypertension, hyperglycemia and overweight), lifestyle (high energy intake and physical inactivity) and endometrial cancer risk in a Norwegian cohort. Int. J Cancer 104, 669-676 (2003).

43. Ollberding, N. J. et al. Legume, soy, tofu, and isoflavone intake and endometrial cancer risk in postmenopausal women in the multiethnic cohort study. J Natl. Cancer Inst. 104, 67-76 (2012).

44. Sponholtz, T. R. et al. Body Size, Metabolic Factors, and Risk of Endometrial Cancer in Black Women. Am. J. Epidemiol. 183, 259-268 (2016)

45. Gelber, R. P., Gaziano, J. M., Manson, J. E., Buring, J. E. \& Sesso, H. D. A prospective study of body mass index and the risk of developing hypertension in men. Am. J. Hypertens. 20, 370-377 (2007).

46. Rossi, R., Turco, V., Origliani, G. \& Modena, M. G. Type 2 diabetes mellitus is a risk factor for the development of hypertension in postmenopausal women. J. Hypertens. 24, 2017-2022 (2006).

47. Moher, D., Liberati, A., Tetzlaff, J. \& Altman, D. G. Preferred reporting items for systematic reviews and meta-analyses: the PRISMA statement. BMJ 339, b2535 (2009).

48. DerSimonian, R. \& Laird, N. Meta-analysis in clinical trials. Control Clin. Trials 7, 177-188 (1986).

49. Hamling, J., Lee, P., Weitkunat, R. \& Ambuhl, M. Facilitating meta-analyses by deriving relative effect and precision estimates for alternative comparisons from a set of estimates presented by exposure level or disease category. Stat. Med. 27, 954-970 (2008).

50. Higgins, J. P. \& Thompson, S. G. Quantifying heterogeneity in a meta-analysis. Stat. Med. 21, 1539-1558 (2002).

51. Egger, M., Davey, S. G., Schneider, M. \& Minder, C. Bias in meta-analysis detected by a simple, graphical test. BMJ 315, 629-634 (1997).

52. Begg, C. B. \& Mazumdar, M. Operating characteristics of a rank correlation test for publication bias. Biometrics 50, 1088-1101 (1994).

53. Wells, G. et al. The Newcastle-Ottawa Scale (NOS) for assessing the quality of nonrandomised studies in meta-analyses. http://www. ohri.ca/programs/clinical_epidemiology/oxford.asp, Accessed 13.08.2014.

54. Bjorge, T. et al. Metabolic syndrome and endometrial carcinoma. Am J Epidemiol. 171, 892-902 (2010).

55. Cust, A. E. et al. Metabolic syndrome, plasma lipid, lipoprotein and glucose levels, and endometrial cancer risk in the European Prospective Investigation into Cancer and Nutrition (EPIC). Endocr. Relat Cancer 14, 755-767 (2007).

56. Settnes, A. \& Jorgensen, T. Hypertension and hysterectomy in Danish women. Obstet. Gynecol. 92, 274-280 (1998).

57. Settnes, A., Andreasen, A. H. \& Jorgensen, T. Hypertension is associated with an increased risk for hysterectomy: a Danish cohort study. Eur. J. Obstet. Gynecol. Reprod. Biol. 122, 218-224 (2005).

58. Radin, R. G. et al. Hypertension and risk of uterine leiomyomata in US black women. Hum. Reprod. 27, 1504-1509 (2012).

59. Benshushan, A., Paltiel, O., Rojansky, N., Brzezinski, A. \& Laufer, N. IUD use and the risk of endometrial cancer. Eur. J. Obstet. Gynecol. Reprod. Biol. 105, 166-169 (2002).

60. Dumesic, D. A. \& Lobo, R. A. Cancer risk and PCOS. Steroids 78, 782-785 (2013).

61. Hamet, P. Cancer and hypertension: a potential for crosstalk? J. Hypertens. 15, 1573-1577 (1997).

62. Bangalore, S. et al. Antihypertensive drugs and risk of cancer: network meta-analyses and trial sequential analyses of 324,168 participants from randomised trials. Lancet Oncol. 12, 65-82 (2011).

63. Pasternak, B., Svanstrom, H., Callreus, T., Melbye, M. \& Hviid, A. Use of angiotensin receptor blockers and the risk of cancer. Circulation 123, 1729-1736 (2011).

64. Behrens, I. et al. Hypertensive disorders of pregnancy and subsequent risk of solid cancer-A nationwide cohort study. Int. J. Cancer 139, 58-64 (2016).

65. Hallum, S., Pinborg, A. \& Kamper-Jorgensen, M. Long-term impact of preeclampsia on maternal endometrial cancer risk. Br. J. Cancer 114, 809-812 (2016).

66. Havulinna, A. S. et al. A blood pressure genetic risk score is a significant predictor of incident cardiovascular events in 32,669 individuals. Hypertension 61, 987-994 (2013).

67. Surendran, P. et al. Trans-ancestry meta-analyses identify rare and common variants associated with blood pressure and hypertension. Nat. Genet. 48, 1151-1161 (2016).

\section{Acknowledgements}

This work has been supported by funding from the Liaison Committee between the Central Norway Regional Health Authority (RHA) and the Norwegian University of Science and Technology (NTNU) and by the Imperial College National Institute of Health Research (NIHR) Biomedical Research Centre (BRC).

\section{Author Contributions}

Conceived and designed the research: D.A. Acquired the data: D.A., A.S. Analyzed and interpreted the data: D.A., A.S., L.J.V. Performed statistical analysis: D.A. Handled funding and supervision: L.J.V. Drafted the manuscript: D.A., A.S., L.J.V. Made critical revision of the manuscript for intellectual content: D.A., A.S., L.J.V. Reference screening: D.A., A.S.

\section{Additional Information}

Supplementary information accompanies this paper at http://www.nature.com/srep

Competing Interests: The authors declare no competing financial interests.

How to cite this article: Aune, D. et al. Hypertension and the risk of endometrial cancer: a systematic review and meta-analysis of case-control and cohort studies. Sci. Rep. 7, 44808; doi: 10.1038/srep44808 (2017). 
Publisher's note: Springer Nature remains neutral with regard to jurisdictional claims in published maps and institutional affiliations.

(c) (i) This work is licensed under a Creative Commons Attribution 4.0 International License. The images or other third party material in this article are included in the article's Creative Commons license, unless indicated otherwise in the credit line; if the material is not included under the Creative Commons license, users will need to obtain permission from the license holder to reproduce the material. To view a copy of this license, visit http://creativecommons.org/licenses/by/4.0/

(C) The Author(s) 2017 


\section{SCIENTIFIC REP:RTS}

OPEN Corrigendum: Hypertension and the risk of endometrial cancer: a systematic review and metaanalysis of case-control and cohort studies

\section{Dagfinn Aune, Abhijit Sen \& Lars J. Vatten}

Correction to: Scientific Reports https://doi.org/10.1038/srep44808; published online 07 April 2017; updated 06 April 2018

In this Article, Abhijit Sen and Lars J. Vatten are incorrectly listed with 'Department of Epidemiology and Biostatistics, Imperial College, London, UK'. The correct affiliation for these authors is listed below:

Department of Public Health and General Practice, Faculty of Medicine, Norwegian University of Science and Technology, Trondheim, Norway.

(c) (i) This work is licensed under a Creative Commons Attribution 4.0 International License. The images or other third party material in this article are included in the article's Creative Commons license, unless indicated otherwise in the credit line; if the material is not included under the Creative Commons license, users will need to obtain permission from the license holder to reproduce the material. To view a copy of this license, visit http://creativecommons.org/licenses/by/4.0/

(C) The Author(s) 2018 